\title{
Canine Thyroid Gland Medullary
} Carcinoma

National Cancer Institute

\section{Source}

National Cancer Institute. Canine Thyroid Gland Medullary Carcinoma. NCI Thesaurus.

Code C161006.

Thyroid gland medullary carcinoma occurring in a dog. 